\title{
Length contrast in the Müller-Lyer figure: Functional equivalence of temporal and spatial separation
}

\author{
KEVIN JORDAN \\ San Jose State University, San Jose, California \\ and \\ JOHN UHLARIK \\ Kansas State University, Manhattan, Kansas
}

\begin{abstract}
The distortion of perceived line length produced by the Müller-Lyer figures reverses from assimilation to contrast under conditions of temporal separation of contextual fins from judged shaft (Experiment 1) and spatial separation of contextual and judged elements (Experiment 2). In both experiments, the outgoing fins produced underestimation of judged shaft length whereas ingoing fins produced overestimation. These findings were predicted by the "pool and store" model of perceptual distortions (Girgus \& Coren, 1982) and point to the need for a unified model of assimilation and contrast of perceived size. Extensions and refinements of the "pool and store" model are proposed.
\end{abstract}

Perceptions of line length are often distorted when a line is presented in the context of other lines. These distortions are labeled assimilation or contrast effects, depending on the direction of distortion. Distortions of perceived length toward the context are assimilation effects; distortions away from the context are contrast effects. Examples of configurations that produce length distortion are the Müller-Lyer and parallel lines arrays. Both examples of length distortion are considered assimilation effects because the perceived length of the judged, or focal, line is typically distorted in the direction of the context relative to a no-context control.

Much research on the Müller-Lyer illusion has investigated the effects of various parametric manipulations, such as fin size and fin angle, on illusion strength (see Coren \& Girgus, 1978, and Robinson, 1972, for reviews). Such studies have usually found changes in the magnitude of assimilation as fin size or angle changed. Additionally, in several of the Müller-Lyer studies reviewed by Day (1972), although contrast was reported in some Müller-Lyer-like figures (e.g., Lewis, 1909, reported length contrast when the contextual "fins" were collapsed to form a straight line continuous with the judged shaft), the usual finding was assimilation. This has led many investigators to classify Müller-Lyer distortions as exclusively assimilation effects, and, as a result, most models of the distortions have been concerned primarily with predicting the magnitude of the assimilation effects (e.g., Erlebacher \& Sekuler, 1969; Pressey, 1972).

The preparation of this report was supported by NASA Grant NCC2-237 to Kevin Jordan. Requests for reprints should be sent to Kevin Jordan, Department of Psychology, San Jose State University, San Jose, CA 95192 .
However, Coren and Girgus (1978; see also Girgus \& Coren, 1982) have recently proposed a unified model of assimilation and contrast of perceived size which specifies the conditions under which either type of distortion can be observed for a wide variety of arrays. According to this "pool-and-store" model, viewing conditions impose constraints on the observer's information processing strategies which result in various perceptual distortions. Simultaneous sampling of both contextual and focal elements in an array results in perceptual "pooling," and hence assimilation, according to the model. On the other hand, sequential processing of contextual and focal elements, which results in the encoding of differences due to the need to "store" the initial elements sampled from the array, results in contrast. This model is concerned with predicting the direction of size distortions (i.e., assimilation or contrast) rather than the magnitude of the distortions.

The pool-and-store model has received support from studies of the parallel lines configuration. For example, using an aftereffect paradigm, Jordan and Uhlarik (1985) reported length contrast when the contextual line in the parallel lines array was inspected for either 5 or $60 \mathrm{sec}$ prior to judgment of the test-line. The temporal separation of contextual and test lines in this paradigm produces sequential processing by definition, and thus the finding of contrast under these conditions is consistent with the "store"' aspect of the model. Additionally, using a standard illusion paradigm involving simultaneous presentation of contextual and focal lines, Jordan and Schiano (1985) have reported length contrast in the parallel lines array with large spatial separation of the contextual line from the focal line. According to Girgus and Coren 
(1982), large spatial separations should lead "to sequential information input of the parts of the array, since the observer apprehends the array in a series of successive fixations over the figure"' (p. 558). Thus, either temporal or spatial separation of contextual and test elements of the parallel lines array produces length contrast rather than assimilation.

The above treatment of the pool-and-store model and the cited empirical findings suggest a functional equivalence of temporal and spatial separation in producing a shift from length assimilation to length contrast. It seems that any experimental manipulation which forces an observer to sequentially process the components of an array should produce contrast. The present experiments were designed to test the applicability of the pool-andstore model to the Müller-Lyer figure. If the pool-andstore model applies to the Müller-Lyer figures, and if similar processes are involved in the production of the parallel lines and Müller-Lyer distortions, the pool-and-store model would predict a reversal from assimilation to contrast of perceived length when the contextual "fins" are either temporally separated (Experiment 1 ) or spatially separated (Experiment 2) from the focal "shaft" in the Müller-Lyer array.

\section{EXPERIMENT 1}

The present experiment was designed to test the prediction of the pool-and-store model that temporal separation of the contextual fins from the focal shaft in the MüllerLyer figure will result in a shift from length assimilation to contrast. In the case of contrast, the outgoing-fins version of the Müller-Lyer array would be expected to produce underestimation of focal shaft length and the ingoing fins would produce overestimation.

There were three conditions in the experiment. The first was simply a standard Müller-Lyer illusion condition involving simultaneous presentation of the fins and shaft for $5 \mathrm{sec}$. This condition was used to demonstrate the usually observed assimilation effect and to serve as a basis for comparison with the conditions involving temporal separation of contextual and focal stimuli. The two latter conditions were considered aftereffect conditions because they involved either 5- or 60-sec exposure to the contextual fins prior to judgment of the focal shaft. Sixty seconds was chosen because this was the duration used by Brigell and Uhlarik (1979) in the initial report of the aftereffect of parallel lines and thus would allow direct comparison of aftereffect data for the Müller-Lyer and parallel lines arrays. Five seconds of prior contextual exposure was chosen as approximately equivalent to the amount of contextual exposure in the simultaneous (illusion) condition. The inclusion of this exposure duration is important because it was an attempt to control a confound between the illusion and aftereffect paradigms. If only $60 \mathrm{sec}$ of prior contextual exposure was used in the present experiment, any observed shift from assimilation to contrast of perceived length could be due either to the mode of presentation of contextual and focal stimuli (simultaneous or sequential) or to differential exposure time to the contextual stimulus $(5$ or $60 \mathrm{sec})$. The comparison of the data for the illusion condition with those of this 5-sec aftereffect condition allows a direct assessment of the effect of temporal separation of contextual and focal stimuli on perceived length by controlling for the potentially confounding effect of contextual exposure duration.

\section{Method}

\section{Observers}

Fifty-four students in an introductory psychology course participated in the experiment in order to earn course credit. Eighteen observers were assigned to each of three conditions (i.e., illusion, 5 -sec aftereffect, and $60-\mathrm{sec}$ aftereffect). The observers were required to have at least $20 / 30$ visual acuity with or without correction. They were run in individual sessions.

\section{Stimuli and Design}

All stimulus lines were made with white tape on a black background. The stimuli were then photographed using high-contrast Kodalith film. The resulting $35-\mathrm{mm}$ slide negatives (black lines on a white background) were projected on the rear of a translucent screen by a Kodak random-access slide projector; presentation was controlled by a projection tachistoscope.

On the basis of Erlebacher and Sekuler's (1969) finding that il lusion magnitude is unchanged as the angle of the fins is varied and the length of the arm is adjusted to maintain a constant tip-totip distance, contextual length in the present study was defined as the distance from the tip of one fin to the tip of the other fin for both ingoing and outgoing versions of the Müller-Lyer figure. Thus, contextual length defined by tip-to-tip distance seems more important than the angle defined by the fins. Focal shaft length was defined as the distance from the apex of one contextual fin to the apex of the other fin. Figure 1 presents a schematic representation of contextual and focal elements employed in the present Müller-Lyer configurations. The specific lengths of the focal line and the contextual fins were chosen to produce maximum over- and underestimation of focal length and to facilitate comparison with the aftereffect of parallel lines (Brigell \& Uhlarik, 1979; Jordan \& Uhlarik, 1985). Nakagawa (1958) reported maximum distortion when each fin was one-third the length of the judged line for both versions of the figure. For the outgoing version, this would result in a ratio of contextual length to judged length (i.e., the "framing ratio") of 5:3; for the ingoing figure, the framing ratio would be $2: 3$. Other estimates of the framing ratios producing maximum distortion vary about these values (see Robinson, 1972). In the present study, the contextual lengths were $1.4,1.9,4.2$, and $5.6 \mathrm{~cm}$, and the focal length was $2.8 \mathrm{~cm}$, resulting in framing ratios of $1: 2,2: 3,3: 2$, and $2: 1$, respectively (cf. Jordan \& Uhlarik, 1985). The two shorter contextual

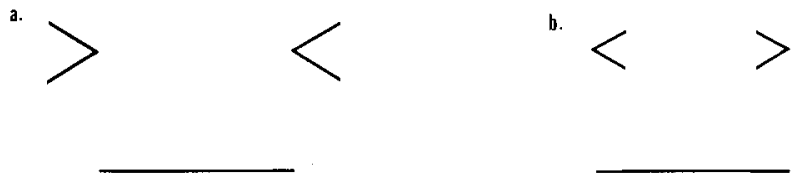

Figure 1. Schematic representation of contextual and focal length in the (a) outgoing and (b) ingoing Müller-Lyer figures used in Experiments 1 and 2 . For both figures, contextual length was defined as the tip-to-tip distance between the fins. Focal shaft length was defined as the distance from apex to apex between fins. The focal shaft was presented subsequent to the contextual fins in the aftereffect conditions of Experiment 1. The shaft and fins were presented simultaneously, but with either a 1.2 or $3.6-\mathrm{cm}$ vertical separation, in Experiment 2. 
lengths $(1.4$ and $1.9 \mathrm{~cm})$ define ingoing figures, and the two longer contextual lengths $(4.2$ and $5.6 \mathrm{~cm})$ define outgoing figures. The angle defined by the fins was $60^{\circ}$, and the focal line was horizontal. The thickness of the lines on the viewing screen was $0.7 \mathrm{~mm}$, and the viewing distance was $77 \mathrm{~cm}$.

In the illusion condition, the $2.8-\mathrm{cm}$ focal line was centered on the viewing screen and the contextual fins were attached to each end of the line. There were four test slides in the illusion condition, one slide each of contextual lengths $1.4,1.9,4.2$, and $5.6 \mathrm{~cm}$, all of which contained the $2.8-\mathrm{cm}$ focal line. The adapting array used in the length aftereffect conditions contained only the fins defining the contextual length. These fins were centered horizontally about a small fixation point in the center of the viewing screen; the focal line was not present in these arrays. Again, the contextual lengths were $1.4,1.9,4.2$, and $5.6 \mathrm{~cm}$ and the subsequently presented focal shaft was $2.8 \mathrm{~cm}$, horizontal, and centered on the viewing screen. To summarize the design of the stimuli, there were three levels of mode of presentation of contextual and focal stimuli (simultaneous, 5 -sec sequential, and 60-sec sequential) and four levels of contextual length $(1.4,1.9,4.2$, and $5.6 \mathrm{~cm})$. The mode of presentation of the contextual and focal stimuli was a between-subjects factor.

Focal judgments were obtained using a graded series comparison scale (cf. Coren \& Girgus, 1972), which was located $9.5 \mathrm{~cm}$ to the right of the midpoint of the focal line. The scale consisted of 11 horizontally oriented lines, labeled "A" through " $\mathrm{K}$ ", which ranged from 2.3 to $3.3 \mathrm{~cm}$ in $1-\mathrm{mm}$ increments. Therefore, the point of objective equality (POE) on this scale for all test stimuli was "F," or $2.8 \mathrm{~cm}$. The lines on the graded series scale were separated vertically by $5 \mathrm{~mm}$. To encourage observers to use a wide range of the response scale, two filler stimuli with focal lengths of 2.4 and $3.2 \mathrm{~cm}$ were included in the design. Responses to these filler stimuli were not included in the data analysis.

To familiarize the observers with the use of the graded series scale, there were also six practice stimuli which consisted of only a focal line. The focal lengths for these stimuli were $2.4,2.6,2.8,2.8$, 3.0 , and $3.2 \mathrm{~cm}$.

\section{Procedure}

The observers viewed the projected image of the stimuli in an otherwise darkened room. The observers were familiarized with the range and the variety of the stimuli, as well as with the use of the graded series scale. Specifically, they were told that the task was to match the apparent length of the focal line to one of the 11 lines on the graded series scale by calling out, to the experimenter, the letter of the matching line. If a focal line appeared intermediate in length to two lines on the graded series scale, the observers were asked to call out the letters for both lines.

Illusion condition. The 18 observers in the illusion condition first proceeded through a random order of the six practice stimuli. These practice trials were followed by a random order of the four test and two filler stimuli. A trial consisted of the simultaneous presentation of the contextual fins and focal line for $5 \mathrm{sec}$, during which the focal judgment was to be made. There was a 10 -sec dark interval separating each trial. After proceeding through the first random order of the test and filler stimuli, the observers proceeded through three more random orders of these six stimuli. In all, each of the 18 observers made 30 focal judgments -6 practice judgments and 4 judgments of each of the four test and two filler stimuli. The entire procedure required approximately $10 \mathrm{~min}$ for each observer.

Aftereffect conditions. The 18 observers in each of the aftereffect conditions also first proceeded through a random order of the six practice stimuli. The observers then proceeded through a random order of the four test and two filler trials. A trial consisted of either a 5 -sec or a 60 -sec inspection of the contextual fins, during which the observer was instructed to fixate the small spot in the center of the adapting array. This adaptation period was followed by the presentation of the focal line for $5 \mathrm{sec}$, during which the length judgment was to be made. The interstimulus interval was $1 \mathrm{sec}$ for both aftereffect conditions. Each trial was separated by a 20 -sec dark interval in order to allow any afterimages to dissipate. Observers then proceeded through three more random orders of the four test and two filler trials with a 2-min break between the second and third random orders. In all, each of these 36 observers made 30 focal judgments -6 practice judgments and 4 judgments of each of the four test and two filler configurations. The procedure required approximately $18 \mathrm{~min}$ for each observer in the 5-sec aftereffect condition and $45 \mathrm{~min}$ for observers in the $60-\mathrm{sec}$ condition.

\section{Results and Discussion}

The responses to the $2.8-\mathrm{cm}$ " no-context" focal line contained in the practice series were averaged to estimate the point of subjective equality (PSE) of the $2.8-\mathrm{cm}$ focal shaft for each group of observers. The mean of the 36 responses ( 2 responses from each of 18 observers) was $2.59 \mathrm{~cm}$ in the illusion condition, $2.61 \mathrm{~cm}$ for the $5-\mathrm{sec}$ aftereffect condition, and $2.62 \mathrm{~cm}$ for the $60 \mathrm{-sec}$ aftereffect condition. This consistent underestimation of objective focal length has been reported by Brigell and Uhlarik (1979) and by Jordan and Uhlarik (1985). Focal judgments were converted into deviations from PSE by subtracting the PSE from the mean judged length of each contextual stimulus for the illusion condition and each of the aftereffect conditions. These data are presented in Figure 2.

Clearly, there was substantial assimilation of focal length in the illusion condition inasmuch as contextual lengths shorter than the $2.8-\mathrm{cm}$ focal shaft (i.e., 1.4 and $1.9 \mathrm{~cm})$ produced underestimation of focal length and lengths longer than the $2.8 \mathrm{~cm}$ focal shaft (i.e., 4.2 and $5.6 \mathrm{~cm}$ ) produced overestimation. This is simply a demonstration of the Müller-Lyer illusion. Additionally, Figure 2 shows that there was contrast of focal length in the aftereffect conditions; contextual lengths shorter than the $2.8-\mathrm{cm}$ focal shaft produced overestimation of focal length, and contextual lengths longer than the $2.8-\mathrm{cm}$ focal shaft produced either underestimation or no distor-

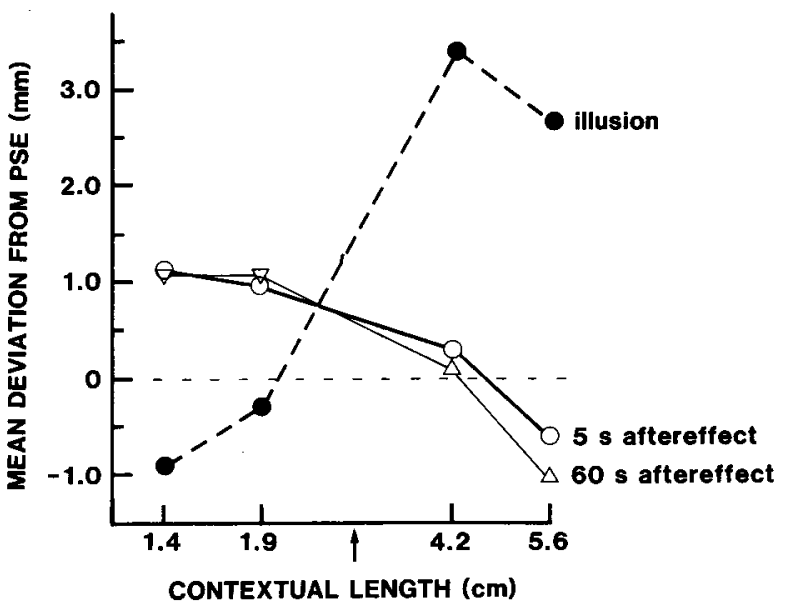

Figure 2. Mean deviations from PSE in millimeters for the illusion, 5-sec aftereffect, and 60-sec aftereffect conditions of Experiment 1. The illusion data indicate assimilation of perceived focal length, and the aftereffect data indicate contrast. The abscissa is a logarithmic scale of length. The arrow on the abscissa indicates the focal shaft length $(2.8 \mathrm{~cm})$ for the test stimuli. 
tion. Finally, in all three curves, the overestimation of perceived length was greater than the underestimation.

The data for the 5- and 60-sec aftereffect conditions were analyzed separately in 4 (contextual lengths) $\times 4$ (replications) $\times 18$ (observers) within-subject analyses of variance. For the 5-sec aftereffect condition, the main effect of contextual length was statistically significant $[F(3,51)=13.47, p<.01]$, which indicates that the length of the contextual fins affected focal judgments. The direction of this effect (see the curve labeled " $5 \mathrm{~s}$ aftereffect" in Figure 2) indicates length contrast. The effect of contextual fin length was also significant in the 60 -sec aftereffect condition $[F(3,51)=28.23, p<.01]$, again indicating that the contextual fins affected focal shaft judgments to produce length contrast. No other main effect or interaction reached statistical significance in either analysis.

There does not appear to be a difference in the magnitude of the length contrast produced by $5-$ or $60-\mathrm{sec}$ inspection of the contextual fins (see Figure 2). This observation was tested by a 2 (inspection durations) $\times 4$ (contextual lengths) $\times 4$ (replications) $\times 18$ (observers) mixed analysis of variance of the data for the 5- and 60sec aftereffect conditions. Inspection duration was a between-subjects factor. The effect of contextual length was again statistically significant $[F(3,102)=40.24, p<$ $.01]$. However, there was no significant effect of inspection duration, indicating that 5 - and $60-\mathrm{sec}$ inspection are equally effective in producing length contrast. This pattern of results is different from that reported by Jordan and Uhlarik (1985) for the parallel lines aftereffect; Jordan and Uhlarik found that $60-\mathrm{sec}$ inspection of a contextual line produced a greater distortion of a focal line than did a 5-sec inspection of the contextual line.

In summary, the results of the present experiment indicate that the mode of presentation (simultaneous or sequential) of the contextual fins and focal shaft in MüllerLyer figures determines whether length assimilation or length contrast occurs. It does not seem to matter how long the contextual fins are inspected; rather, temporal separation of fins and shaft produces contrast and simultaneous viewing produces assimilation.

\section{EXPERIMENT 2}

The pool-and-store model predicts contrast whenever observers are forced to sample an array in multiple glances. Experiment 1 demonstrated length contrast in the Müller-Lyer figure using temporal separation of shaft and fins to produce sequential sampling of the array. Spatial separation of shaft and fins is also expected to produce contrast and, in fact, Coren and Girgus (1978) cite the results of Fellows (1967) as a demonstration of just that phenomenon. Fellows reported a "reverse Müller-Lyer illusion" when the fins of a horizontally oriented MüllerLyer figure were horizontally separated from the focal shaft, producing a gap between shaft and fin. However, it should be noted that this "reversal" was reported only for ingoing fins and, in fact, this finding is best interpreted as length assimilation rather than length contrast. When the fins of Fellows's figure were moved away from the shaft, the net effect was to produce a contextual length that was longer than the focal shaft, and it is therefore not surprising that overestimation of shaft length was observed even when the fins were ingoing. Thus, the apparent contrast of shaft length could have been due to either the spatial separation or to the increase in the contextual length, as defined by the fins, from shorter than to longer than shaft length. Pressey and associates (Pressey \& Bross, 1973; Pressey, DiLollo, \& Tait, 1977) report a similar reversal in the Müller-Lyer illusion with a gap introduced between shaft and fins, and they too argue that the effect is best interpreted as assimilation.

The present experiment represents an attempt to demonstrate length contrast in the Müller-Lyer figure when all parts of the figure are presented simultaneously but with a spatial separation. The confound of contextual length with spatial separation present in the Fellows studies is eliminated by introducing a vertical spatial separation of the contextual fins from a horizontally oriented focal shaft. Under these conditions, contextual length is unchanged despite the introduction of the spatial separation, and therefore any reversal from underestimation to overestimation of focal shaft length produced by ingoing fins could unambiguously be attributed to spatial separation. This manipulation allows for a test of whether spatial separation, per se, produces "storing" and, hence, contrast, as the pool-and-store model predicts.

Girgus and Coren (1982) propose that perceptual "pooling"' occurs with configurations in which the separations among contours are subfoveal. On the other hand, suprafoveal intercontour distances should lead to sequential sampling of the elements of the array and hence "storing." Thus, assimilation should occur with subfoveal intercontour separations, whereas contrast should occur with suprafoveal separations. Precise definition of the size of the fovea has proven difficult, but Ditchburn (1973) defines the fovea as $2^{\circ}$ of visual angle and the parafovea as the area from $2^{\circ}$ to $10^{\circ}$. There were two levels of spatial separation in the present experiment, one subfoveal and the other para- or suprafoveal, and these were compared with the illusion data from Experiment 1 which can actually be considered a third level of spatial separation, $0 \mathrm{~cm}$. The pool-and-store model predicts a reversal from assimilation to contrast with the large, suprafoveal, separation. Thus, ingoing fins that are spatially separated by a suprafoveal intercontour distance from the focal shaft would be expected to produce overestimation of shaft length, and outgoing fins would be expected to produce underestimation.

\section{Method}

\section{Observers}

Thirty-six students in an introductory psychology course participated in the experiment in order to earn course credit. Eighteen observers were assigned to each of the two levels of spatial separa- 
tion. The observers were required to have at least $20 / 30$ visual acuity with or without correction. They were run in individual sessions.

\section{Stimuli and Design}

The stimuli were constructed, photographed, and presented to the observers in the same manner as in Experiment 1.

Contextual length was again defined as the distance from the tip of one fin to the tip of the other fin, and the angle defined by the fins was $60^{\circ}$. The same four contextual lengths $(1.4,1.9,4.2$, and $5.6 \mathrm{~cm}$ ) were used as in the previous experiment, and the focal shaft was $2.8 \mathrm{~cm}$. Thus, the framing ratios (contextual length/focal length) were $1: 2,2: 3,3: 2$, and $2: 1$.

A schematic representation of a test stimulus containing both the contextual fins and the focal shaft is shown in Figure 1. Spatial separation of contextual and focal elements was defined as the vertical distance from the apex of the left contextual fin (for example) to the leftmost point (for example) of the horizontal focal shaft; the separation was defined orthogonally to the focal shaft. Two levels of spatial separation were used, 1.2 and $3.6 \mathrm{~cm}$. At the $77-\mathrm{cm}$ viewing distance, the visual angles of these spatial separations were $0.9^{\circ}$ and $2.7^{\circ}$, respectively. The $1.2-\mathrm{cm}$ spatial separation should produce an array in which most of all three elements in the test array (two contextual fins and the focal shaft) are easily imaged foveally; the 3.6-cm spatial separation should necessitate sequential sampling of the array. Thus, there were two levels of spatial separation (1.2 and $3.6 \mathrm{~cm})$ and four levels of contextual length $(1.4,1.9,4.2$, and $5.6 \mathrm{~cm}$ ). Spatial separation was a between-subjects factor.

Focal judgments were obtained using the same graded series comparison scale used in Experiment 1. Again, to encourage observers to use a wide range of the response scale, two filler stimuli with focal shaft lengths of 2.4 and $3.2 \mathrm{~cm}$ were included for each level of spatial separation. The contextual length defined by the fins was determined randomly for these filler stimuli. Responses to the filler stimuli were not included in the data analysis. Finally, there were six practice stimuli which consisted of only a focal shaft. The lengths of these shafts were $2.4,2.6,2.8,2.8,3.0$, and $3.2 \mathrm{~cm}$.

\section{Procedure}

The procedure for the present experiment was identical to the procedure for the illusion condition of Experiment 1 . The $18 \mathrm{ob}-$ servers in each of the spatial separation conditions proceeded through a random order of the six practice stimuli followed by four random orders of the four test and two filler stimuli. A trial consisted of the simultaneous presentation of the focal shaft and spatially displaced contextual fins for $5 \mathrm{sec}$, during which the focal judgment was to be made. There was a 10 -sec dark interval separating each trial. In all, each observer made 30 focal judgments -6 practice judgments and 4 judgments of each of the four test and two filler stimuli. The entire procedure required approximately $10 \mathrm{~min}$ for each observer.

\section{Results and Discussion}

The responses to the 2.8-cm "no-context" focal shaft contained in the practice series were averaged to estimate the PSE of the 2.8-cm focal shaft for each group of observers. The mean of the 36 responses ( 2 responses from each of 18 observers) was $2.61 \mathrm{~cm}$ for the $1.2-\mathrm{cm}$ spatial separation condition and $2.57 \mathrm{~cm}$ for the $3.6-\mathrm{cm}$ spatial separation condition. This consistent underestimation of the 2.8-cm objective focal length was also reported in Experiment 1 . Focal judgments were converted into deviations from PSE by subtracting the PSE from the mean judged length of each test stimulus for each level of spatial separation. The results of spatially separating the contextual fins from the focal shaft in the Müller-Lyer figure are presented in Figure 3. Also included in Figure 3 for

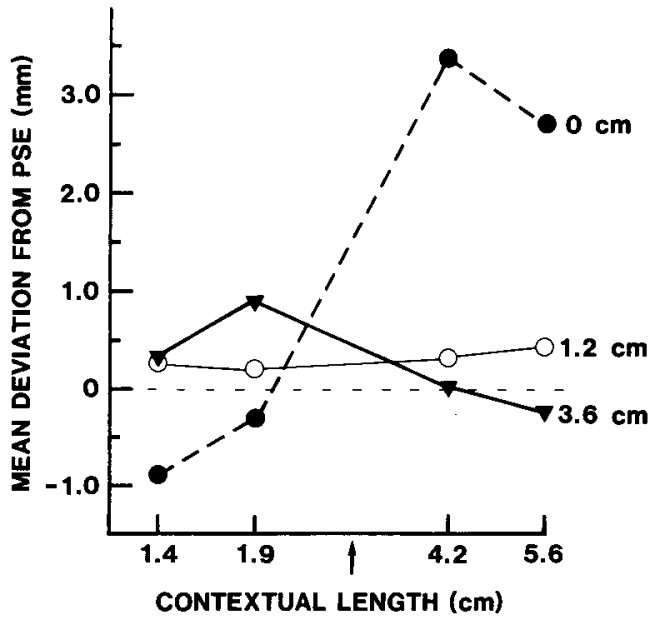

Figure 3. Mean deviations from PSE in millimeters for the 1.2and 3.6-cm spatial separation conditions of Experiment 2. Included for comparison are the illusion data from Experiment 1, which are labeled " $0 \mathrm{~cm}$ " spatial separation. The illusion data indicate assimilation of perceived shaft length, and the data for the $3.6-\mathrm{cm}$ spatial separation condition indicate length contrast. The abscissa is a logarithmic scale of length. The arrow on the abscissa indicates the focal shaft length $(2.8 \mathrm{~cm})$ for the test stimuli.

comparison are the illusion data from Experiment 1, which are labeled as having $0 \mathrm{~cm}$ spatial separation.

The data for the 1.2- and 3.6-cm spatial separation conditions were analyzed separately in 4 (contextual lengths) $\times 4$ (replications) $\times 18$ (observers) within-subject analyses of variance. For the $1.2-\mathrm{cm}$ condition, the main effect of contextual length was not statistically significant, indicating that variations in contextual length did not affect focal shaft judgments differentially; neither assimilation nor contrast occurred. However, the main effect of contextual length was significant in the 3.6-cm separation condition $[F(3,51)=8.43, p<.01]$, indicating a differential effect of contextual length on focal judgments. The direction of this effect (see the curve labeled "3.6 cm" in Figure 3) indicates a truly "reversed MüllerLyer illusion," and hence length contrast; short or ingoing contextual lengths produced overestimation of focal shaft length, and long or outgoing contexts produced some slight underestimation. Again, the magnitude of the overestimation of focal shaft length was greater than the underestimation. No other main effect or interaction reached significance in either analysis.

The results of the second experiment are also generally in agreement with the pool-and-store model. Large, suprafoveal, spatial separations of contextual fins from the focal shaft in the Müller-Lyer figure result in length contrast. By removing the confound of spatial separation and contextual length present in previous research (Fellows, 1967; Pressey \& Bross, 1973), the present experiment demonstrated the importance of spatial separation, per se, in reversing the Müller-Lyer illusion from an assimilation effect to a contrast effect. One puzzling aspect of the present data is the lack of length assimilation in 
the $1.2-\mathrm{cm}$ subfoveal intercontour separation condition. The pool-and-store model predicts assimilation under these conditions, yet no distortion was observed. Perhaps contextual and focal elements in an array are "pooled" more or less on the basis of their proximity; very small intercontour separations might be "pooled" more readily than slightly larger but still subfoveal separations.

\section{GENERAL DISCUSSION}

There are three important results in the present research. First, temporal separation of the contextual fins from the focal shaft in the Müller-Lyer figure produces length contrast. Second, spatial separation of the contextual fins from the focal shaft also produces length contrast in the MüllerLyer figure. Finally, the overestimation of focal shaft length produced by the ingoing fins was greater than the underestimation produced by the outgoing fins for both the sequential and simultaneous length contrasts. Each of these results will be discussed in turn.

\section{Sequential Length Contrast}

Experiment 1 demonstrated that under the appropriate conditions, a Müller-Lyer aftereffect occurs. The effect was the reverse of the usually observed distortion; ingoing fins viewed for either 5 or $60 \mathrm{sec}$ prior to a focal shaft judgment produced overestimation, whereas outgoing fins produced slight underestimation. A similar length contrast effect was reported by Pollack (1964), who had 8to 11 -year-old children judge the focal shaft after 500 msec inspection of outgoing fins of the Müller-Lyer figure. These findings, taken together, support Coren and Girgus's (1978) pool-and-store model of assimilation and contrast. As pointed out in the introduction, the model predicts that any experimental manipulation that encourages observers to sample a visual array in multiple glances should produce contrast due to the need to store the first elements sampled from the array.

The findings of length contrast with $500 \mathrm{msec}, 5 \mathrm{sec}$, and $60 \mathrm{sec}$ of prior contextual exposure point to the problems of any model that proposes that sequential contrast effects are the result of fatigue due to prolonged stimulation of feature-specific neural units (cf. Coltheart, 1971) or lateral inhibition among feature-specific units (Magnussen \& Kurtenbach, 1980). It is difficult to describe how the fatigue or inhibition builds up in either $500 \mathrm{msec}$ or $5 \mathrm{sec}$ in a sequential viewing paradigm but not in $5 \mathrm{sec}$ in a simultaneous viewing (illusion) paradigm. A more parsimonious description of the length contrast is that it results from temporal separation of contextual and focal elements rather than from prolonged exposure to contextual elements.

\section{Simultaneous Length Contrast}

Experiment 2 demonstrated length contrast in the Müller-Lyer figure produced solely by spatial separation of the contextual fins from the focal shaft. As pointed out in the introduction to Experiment 2, previous reports of a similar reversal of the usual Müller-Lyer distortions (Fellows, 1967; Pressey \& Bross, 1973; Pressey et al., 1977) were equivocal in addressing length contrast due to a confound of spatial separation with overall contextual length. Our Experiment 2 removed the confound by varying spatial separation of the contextual fins from the focal shaft while holding contextual length constant. Under these conditions, large suprafoveal spatial separations of contextual and focal elements produced length contrast. This finding is also supportive of the pool-and-store model in that suprafoveal separations would require an observer to sequentially sample the contextual and focal elements. These first two findings, simultaneous and sequential length contrast, indicate a functional equivalence of temporal and spatial separation in producing perceptual distortions.

\section{Asymmetry in the Müller-Lyer Distortions}

One of the most robust findings with the traditional (fins attached) Müller-Lyer figure has been that the distortion produced by the outgoing fins is much greater than that produced by the ingoing fins (see Adam \& Bateman, 1983; see also the curve labeled "illusion" in Figure 2). Additionally, manipulation of one parameter (e.g., the angle formed by the contextual fins) often differentially affects the magnitude of the distortions produced by the ingoing and outgoing versions of the Müller-Lyer figure. This has led some authors to propose that the two illusions are produced by fundamentally different processes (Adam \& Bateman, 1983; Day, 1972; Sekuler \& Erlebacher, 1971) and that the differential magnitudes are somehow intrinsic to the direction of the fins. If this were true, outgoing fins should have produced larger distortions than ingoing fins in the present research. This, of course, did not occur. In both experiments, there was a reversal of the asymmetry; the ingoing fins produced larger distortions than the outgoing fins.

An alternative approach to asymmetries in length distortions (Brigell, Uhlarik, \& Goldhorn, 1977) proposes that similar processes underlie overestimation and underestimation, but that the asymmetry results from logarithmic representation of length in the visual system. According to this proposal, overestimation of length will always be larger than underestimation, since a linear shift up on a logarithmic scale will be larger than an equal linear shift down the scale. This is consistent with the present results as well as with data obtained with the parallel lines figure (Jordan \& Uhlarik, 1985). Therefore, the present results suggest that the distortions of perceived focal shaft length produced by outgoing and ingoing fins in the Müller-Lyer figure are produced by the same pool-andstore processing strategy regardless of whether assimilation or contrast occurs and of whether over- or underestimation occurs.

\section{Conclusions}

The present research indicates that any model of the Müller-Lyer illusion must be able to account for both as- 
similation and contrast distortions. Furthermore, any model of the Müller-Lyer illusion must be able to make a priori statements of new phenomena derivable from the model. The pool-and-store model does well on both of these criteria; the model accounts for both assimilation and contrast and predicted the two new phenomena reported here. In applying the model to the Müller-Lyer illusion, it is clear that there is nothing intrinsic to the contextual fins in producing the distortions. Consistent with this statement are the numerous demonstrations that just about anything connected to a focal shaft (e.g., circles or arcs) will produce distortions similar to the MüllerLyer illusion (Coren \& Girgus, 1978; Robinson, 1972). The critical variables determining length assimilation and contrast are the spatial and temporal proximity of contextual and focal stimuli. These variables allow for a simple classification system for distortions of perceived length produced by the Müller-Lyer and parallel lines figures (cf. Girgus \& Coren, 1982). Length assimilation results when the contextual and focal stimuli are presented simultaneously and in close spatial proximity. Conversely, length contrast is produced either by sequential presentation of contextual and focal stimuli or by the simultaneous presentation of contextual and focal stimuli with a large spatial separation. Whether over- or underestimation of perceived length occurs simply depends on the relationship between contextual length and focal length.

There seem to be three basic tasks pursuant to the refinement of this pool-and-store classification system. First, the system outlined above should be applied to other distortions of linear extent, such as the Baldwin illusion, to test its generalizability. As noted previously, Jordan and Uhlarik (1985) have demonstrated that temporal separation, per se, produces length contrast in the parallel lines figure, and Jordan and Schiano (1985) reported length contrast produced by spatial separation.

The second task in the refinement of the pool-and-store model is to provide for a specification of the predicted magnitude of the assimilation or contrast effects. As noted previously, the model is more concerned, in its present form, with the specification of the direction of the distortions than with their magnitude. Pressey and his associates (Pressey, 1972; Pressey \& Murray, 1976) have stated several postulates in assimilation theory which do a good job in estimating the magnitude of length assimilation in a variety of illusion configurations. Perhaps these postulates could be extended or revised to include length contrast effects and incorporated into the pool-and-store model.

Finally, the model has to deal more specifically with the criterion for distinguishing "small" from "large" spatial separations; that is, the model must state when pooling will occur and when storing will occur. It seems that the statement of the subfoveal-suprafoveal criterion (Girgus \& Coren, 1982) is specific and testable. However, there is no consensus among visual scientists on the dimensions of the fovea. An additional possibility is that distinguishing small and large (pooling and storing) might not have as much to do with absolute measures of spatial separation as Girgus and Coren suggest. Rather, large spatial separations might be defined relative to the size of the focal or judged line. In fact, Pressey and DiLollo (1978) state that length assimilation will occur when both contextual and focal elements are within an "attentive field." Although the operational specification of the attentive field has proven to be one of the major problems of assimilation theory (Bross, Blair, \& Longtin, 1978; Pressey, 1979), Pressey and DiLollo suggest that the attentive field size changes as a ratio of focal size. It could be that those stimuli within an attentive field are perceptually pooled, and hence assimilated, and those outside the attentive field are stored, with contrast the result. The suprafoveal spatial separation that produced length contrast in Experiment 2 might have actually resulted in the contextual fins' being outside the attentive field. It should certainly be possible to determine the involvement of absolute and relative spatial separation in the shift from length assimilation to contrast.

\section{REFERENCES}

ADAM, J., \& BATEMAN, L. (1983). A correlational analysis of symmetry between the arrowhead and featherhead Mueller-Lyer illusions. Perception, 12, 119-129.

BRIGELL, M., \& UhLARIK, J. (1979). The relational determination of length illusions and length aftereffects. Perception, 8, 187-197.

Brigell, M., Uhlarik, J., \& GoldhorN, P. (1977). Contextual influences on judgments of linear extent. Journal of Experimental Psychology: Human Perception \& Performance, 3, 105-118.

Bross, M., Blair, R., \& Longtin, P. (1978). Assimilation theory, attentive fields and the Mueller-Lyer illusion. Perception, 7, 297-304.

Coltheart, M. (1971). Visual feature-analyzers and aftereffects of tilt and curvature. Psychological Review, 78, 114-121.

Coren, S., \& Girgus, J. S. (1972). A comparison of five methods of illusion measurement. Behavior Research Methods \& Instrumentation, 4, 240-244.

COREN, S., \& Girgus, J. S. (1978). Seeing is deceiving: The psychology of visual illusions. Hillsdale, NJ: Erlbaum.

DAY, R. H. (1972). Visual spatial illusions: A general explanation. Science, 175, 1335-1340.

DITCHBURN, R. W. (1973). Eye-movements and visual perception. Oxford: Clarendon Press.

ERLebacher, A., \& SekUler, R. (1969). Explanation of the MuellerLyer illusion: Confusion theory examined. Journal of Experimental Psychology, 80, 462-467.

Fellows, B. J. (1967). Reversal of the Mueller-Lyer illusion with changes in length of the interfins line. Quarterly Journal of Psychology, 19, 208-214.

GiRGUS, J. S., \& CoReN, S. (1982). Assimilation and contrast illusions: Differences in plasticity. Perception \& Psychophysics, 32, 555-561.

JoRDan, K., \& Schiano, D. J. (1985, April). Serial processing and the parallel line illusion: Contrast through spatial separation. Paper presented at the meeting of the Western Psychological Association, San Jose, CA.

JoRDAN, K., \& UhlaRIK, J. (1985). Assimilation and contrast of perceived length depend on temporal factors. Perception \& Psychophysics, 37, 447-454.

LEWIS, E. O. (1909). Confluxion and contrast effects in the MuellerLyer illusion. British Journal of Psychology, 3, 21-41.

Magnussen, S., \& Kurtenbach, W. (1980). Adapting to two orientations: Disinhibition in a visual aftereffect. Science, 207, 908-909.

Nakagawa, D. (1958). Mueller-Lyer illusion and retinal induction. Psychologia, 1, 167-174. 
Pollack, R. H. (1964). Simultaneous and successive presentation of the Mueller-Lyer figure and chronological age. Perceptual \& Motor Skills, 19, 303-310.

PREsSEY, A. W. (1972). The assimilation theory of geometric illusions: An additional postulate. Perception \& Psychophysics, 11, 28-30.

Pressey, A. W. (1979). Are black circles attentive fields? A reply to Bross, Blair, and Longtin. Perception, 8, 237-238.

Pressey, A. W., \& Bross, M. (1973). Assimilation theory and the reversed Mueller-Lyer illusion. Perception, 2, 211-217.

Pressey, A. W., \& Dilollo, V. (1978). Effects of distance between standard and comparison lines on the Mueller-Lyer illusion. Perception \& Psychophysics, 24, 415-419.

Pressey, A. W., Dilollo, V., \& Tait, R. W. (1977). Effects of gap size between shaft and fins and of angle of fins on the Mueller-Lyer illusion. Perception, 6, 435-439.

Pressey, A. W., \& Murray, R. (1976). Further developments in the assimilation theory: The adjacency principle. Perception \& Psychophysics, 19, 536-544.

Robinson, J. O. (1972). The psychology of visual illusion. London: Hutchinson.

Sekuler, R., \& Erlebacher, A. (1971). The two illusions of MuellerLyer: Confusion theory reexamined. American Journal of Psychology, 84, 477-481.

(Manuscript received September 18, 1985; revision accepted for publication April 2, 1986.) 